\title{
A randomised, double-blind, cross-over trial to evaluate bread, in which gluten has been pre-digested by prolyl endoprotease treatment, in subjects self-reporting benefits of adopting a gluten-free or low-gluten diet
}

\author{
Dinka Rees ${ }^{1}$, Grietje Holtrop ${ }^{2}$, Gemma Chope ${ }^{3}$, Kim M. Moar ${ }^{1}$, Morven Cruickshank ${ }^{1}$ and \\ Nigel Hoggard ${ }^{1 *}$ \\ ${ }^{1}$ Rowett Institute, University of Aberdeen, Foresterhill AB25 2ZD, UK \\ ${ }^{2}$ Biomathematics and Statistics Scotland, Aberdeen, Foresterbill AB25 2ZD, UK \\ ${ }^{3}$ Campden BRI, Chipping Campden, Gloucestershire GL55 6LD, UK \\ (Submitted 10 May 2017 - Final revision received 30 November 2017 - Accepted 12 December 2017)
}

\section{Abstract}

The aim of the present study was to determine if the enzyme Aspergillus niger prolyl endoprotease (ANPEP), which degrades the immunogenic proline-rich residues in gluten peptides, can be used in the development of new wheat products, suitable for gluten-sensitive (GS) individuals. We have carried out a double-blind, randomised, cross-over trial with two groups of adults; subjects, self-reporting benefits of adopting a gluten-free or low-gluten diet (GS, $n$ 16) and a control non-GS group ( $n$ 12). For the trial, volunteers consumed four wheat breads: normal bread, bread treated with 0.8 or $1 \%$ ANPEP and low-protein bread made from biscuit flour. Compared with controls, GS subjects had a favourable cardiovascular lipid

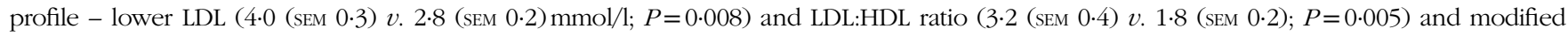
haematological profile. The majority of the GS subjects followed a low-gluten lifestyle, which helps to reduce the gastrointestinal (GI) symptoms severity. The low-gluten lifestyle does not have any effect on the quality of life, fatigue or mental state of this population. Consumption of normal wheat bread increased GI symptoms in GS subjects compared with their habitual diet. ANPEP lowered the immunogenic gluten in the treated bread by approximately $40 \%$. However, when compared with the control bread for inducing GI symptoms, no treatment effects were apparent. ANPEP can be applied in the production of bread with taste, texture and appearance comparable with standard bread.

Key words: Gluten sensitivity: Gastrointestinal symptoms: Lipid profile: Enzymes: Aspergillus niger prolyl endoprotease

Coeliac disease is an autoimmune response to the major wheat protein, gluten and affects approximately one in a hundred individuals. However, general practitioners and gastroenterologists are encountering patients claiming gluten-related symptoms without a definitive diagnosis of coeliac disease. These individuals have been defined as suffering from gluten sensitivity ${ }^{(1)}$. The diagnosis of non-coeliac gluten-sensitive (NCGS) patients is difficult, as is the management, because there are no known biomarkers. It has been reported that gluten-free diet (GFD) alleviates symptoms in subjects with the condition $^{(1)}$. However, NCGS is not well characterised at present and there is only a limited consensus on its presence and diagnosis $^{(2)}$. It is also not clear what exactly are the dietary requirements for this population and whether the affected individuals can tolerate low doses of gluten as opposed to following a strict GFD. As the majority of the gluten-sensitive (GS) subjects are self-diagnosed, information is scarce on whether their gluten intake is any different from that of the general or coeliac population. Therefore, further characterisation of subjects claiming sensitivity to gluten is important to better understand the aetiology of the syndrome and to ensure that the dietary needs of people with the condition are adequately met.

Gluten is a generic term for a family of proteins which include polymeric (larger molecular weight) glutenins and monomeric (lower molecular weight) gliadins. There is evidence that the ingestion of gluten plays a part in the aetiology of coeliac disease, inducing cell-mediated immunological responses in the small intestine and flattening of the mucosa ${ }^{(3)}$. NCGS lacks the characteristic serological and histological markers of coeliac disease $^{(4,5)}$, suggesting different patho-physiological mechanisms. In addition, the diet component(s) involved in the pathogenesis of NCGS have not as yet been identified. However, it is suspected that gluten is a candidate, although there are some studies showing that Fermentable, Oligo-, Di-, Mono-saccharides and Polyols (FODMAP) might be the triggering factors ${ }^{(6)}$. Other

Abbreviations: ANPEP, Aspergillus niger prolyl endoprotease; CD, coeliac disease; FODMAP, Fermentable, Oligo-, Di-, Mono-saccharides and Polyols; GFD, gluten-free diet; GI, gastrointestinal; GS, gluten sensitive; NCGS, non-coeliac gluten sensitive; SF-36, 36-Item Short Form Health Survey; VAS, visual analogue scale.

* Corresponding author: N. Hoggard, email n.hoggard@abdn.ac.uk 
studies however report gluten being the culprit in NCGS individuals, in whom FODMAP intolerances have been ruled out ${ }^{(7)}$. More resent evidence also suggests that members of the nongluten $\alpha$-amylase/trypsin inhibitor (ATI) family contained in wheat $^{(8)}$ may play a role.

As the products for customers with gluten-related disorders are often reported to be unsatisfactory in terms of palatability, the manufacturers are seeking to develop new gluten-free or gluten-detoxified alternatives. One approach, which has received consideration, is the use of enzymes directed against the immunogenic proline rich residues in gluten peptides ${ }^{(9)}$. Gluten is especially rich in proline, which renders the immunogenic epitopes resistant to gastrointestinal (GI) digestion $^{(10,11)}$. It has been reported that in vitro the enzyme Aspergillus niger prolyl endoprotease (ANPEP), produced from the fungus Aspergillus niger, is capable of degrading intact gluten molecules and $\mathrm{T}$ cell stimulatory epitopes from gluten into non-immunogenic, harmless fragments ${ }^{(12)}$. ANPEP is also highly efficient in accelerating the degradation of both gliadin and glutenin molecules in a GI model system and in the stomach of healthy volunteers ${ }^{(13,14)}$. However, one problem in the use of enzymes such as ANPEP in baking is that they target the digestion of gluten, which confers the unique visco-elastic properties of the dough that allows it to be processed into bread. Thus, we needed to determine if ANPEP could be used at levels, which rendered the gluten harmless, but could be used in the production of wheat products, comparable with standard gluten content products in terms of taste, texture and appearance.

In this study we have investigated if ANPEP-treated bread is suitable for consumption by subjects self-reporting benefits of adopting a low-gluten or GFD. In addition, we have characterised this group of subjects by comparing them with a non-GS control group.

\section{Methods \\ Experimental bread}

Four types of white bread were produced (Warburton Ltd.), broadly following the Chorleywood bread process ${ }^{(15)}$. The four variants were as follows: a reference/control bread (the flour used was a bread-making grist of $11.9 \%$ protein at $14 \%$ moisture); a protease-treated bread (treated with $0.8 \%$ ANPEP); a second protease-treated bread (treated with 1\% ANPEP); and a lowerprotein bread made from biscuit flour $(9.2 \%$ protein at $14 \%$ moisture). These four treatments allowed us to investigate the responses of self-defined GS individuals to breads with decreasing gluten content. The protease ANPEP was provided by DSM Food Specialities B.V. at an activity of 43 propyl peptidase units/g (WO2005/027953). Other additions, such as salt, yeast and minor processing additives were constant throughout and in accordance with standard commercial practice however, certain minor adjustments were made to the addition of water in the dough to allow the breads to be processed correctly.

The experimental bread was delivered sliced $(35 \mathrm{~g} /$ slice), with the crusts removed from each loaf. It was packed in transparent bags, labelled as bread A, B, C or D. The code was held by an independent observer. In a preliminary test, ten healthy individuals were unable to detect any differences in the taste, smell and appearance of the four breads. The bread was kept frozen until required.

The proteins of the bread were measured by the Dumas combustion method, using Leco FP528 protein analyser (LECO) according to accepted standards and by Gluten-Tec ${ }^{\circledR}$ ELISA (EuroProxima) for quantitative detection of $\alpha$-20 gliadin and gliadin fragments in food and competitive RIDASCREEN ${ }^{\circledR}$ Gliadin ELISA (R-Biopharm AG) for analysis of R5 epitope. In the case of the R5 ELISA (lower limit of quantification $10 \mathrm{mg}$ gluten/kg and reproducibility around $37 \%$ ), gliadin was extracted by the universal prolamin and glutelin extractant solution procedure of Mena et al. ${ }^{(16)}$, whereas in the case of the $\alpha$-20 ELISA (lower limit of quantification $153 \mu \mathrm{g}$ peptide/kg and reproducibility for incurred baked matrices of around $45 \%$ ), gluten extraction used 60\%-ethanol-dithiothreitol according to the manufacturers' instructions.

\section{Subjects}

This study was conducted according to the guidelines laid down in the Declaration of Helsinki and all procedures involving human subjects were approved by the Rowett Institute Ethics Committee in April 2014. Recruitment was through a variety of sources including press releases, radio adverts, newspaper adverts, posters, flyers and presentations to the public. Written informed consent was obtained from all subjects. The study took place between June 2014 and June 2015. Adults from two groups were invited to participate - those who believed they experience sensitivity to gluten, but were willing to consume breads made of wheat flour (including normal gluten containing bread) and healthy volunteers, who acted as a control group.

A total of 153 subjects expressed interest in the study however, many of these declined to participate in the trial as they were following a strict GFD and were not prepared to undergo gluten rechallenge. The recruitment process is illustrated in Fig. 1. Those subjects, who were willing to continue with the trial ( $n$ 62), were invited to complete a health and lifestyle questionnaire (screening visit) to assess their general health and their sensitivity to gluten/wheat. For the evaluation of dietary compliance with the GFD, a verified flow chart ${ }^{(17,18)}$ was used. This numerical five-level score system is based on strategies that subjects use to avoid eating gluten ${ }^{(17)}$. Initial investigation for coeliac disease was through a questionnaire incorporating the following questions: 'Have you ever been tested for coeliac disease? If yes, how long ago was the test done?'; 'Did you have a blood test for coeliac disease? Was the blood test: positive; negative; I do not know'; 'Did you have a duodenal biopsy? Was the biopsy: positive; negative; I do not know'. All volunteers who reported a diagnosis of coeliac disease (CD) were excluded. Those volunteers who completed tests for CD (duodenal biopsy or immunological tests) but the CD diagnosis was not confirmed were invited to participate in the study. These volunteers believed that avoiding gluten helped to reduce their discomfort. Volunteers were also excluded if they had been previously diagnosed with dermatitis herpetiformis, wheat allergy or GI disorders; were unable to give written informed consent; were on medications for treatment of GI 
symptoms or were taking digestive enzymes; were pregnant/ breast-feeding; had poorly controlled psychiatric disease or had any other significant health issue. A total of thirty-two subjects were enrolled in the study and were randomly allocated by an independent statistician in a double-blind, cross-over fashion to the treatments (breads). The volunteers were invited to complete the Quality of Life (36-Item Short Form Health Survey $(\text { SF-36) })^{(19)}$, Physical Activity (Beacke $)^{(20)}$ and Depression, Anxiety and Stress (DASS 21) ${ }^{(21)}$ questionnaires, whereas on their habitual diet and before the interventions began. In all, twenty-eight subjects (twenty female and eight male) completed the trial (sixteen self-reported sensitive to gluten subjects and twelve healthy volunteers). The reasons for drop-outs were as follows: reappearance of severe GI symptoms (scoring above 80 on a $100 \mathrm{~mm}$ visual analogue scale (VAS)) while on trial breads (two GS volunteers); non-compliance with study diaries (one GS volunteer); and noncompliance with bread consumption (one GS volunteer).

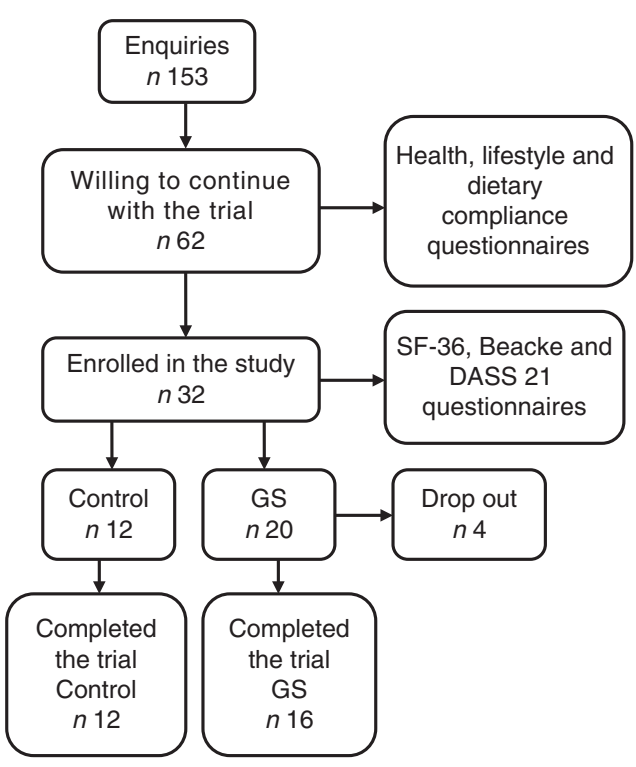

Fig. 1. Study recruitment. SF-36, 36-Item Short Form Health Survey, DASS 21, Depression, Anxiety and Stress Scale; GS, gluten sensitive.

\section{Study design}

Blood samples from both, control and GS groups, were collected between 07.00 and 10.00 hours after 10 plus hours of fasting before the interventions began, using serum-, EDTA- or heparinised- evacuated tubes (BD Vacutainer ${ }^{\circledR}$ tubes; Becton Dickinson UK Ltd). Serum and plasma were stored at $-70^{\circ} \mathrm{C}$ until they were analysed.

After 1 week on their habitual diet (week 0), subjects were randomly allocated in a double-blind, cross-over fashion to one of the four breads (Fig. 2). The trial participants were instructed to consume each treatment bread for 1 week (3 slices/d $105 \mathrm{~g}$ ), replacing the bread they normally ate with the experimental bread. For the duration of the study, the volunteers were asked to continue to eat their habitual diet, but to refrain from eating gluten containing foods (as well as other foods), which they considered to cause GI discomfort. The experimental period was followed by a 14-d wash-out period before crossing over to the next bread. Subjects experiencing reappearance of intolerable symptoms on a treatment bread were asked to cease the study bread of that particular intervention but to continue to fill in the GI symptoms and daily fatigue diaries. Subjects were allowed to continue with the trial if they wished. Compliance was assessed verbally at regular reviews and counting the slices of bread left at the end of the treatment week.

\section{Gastrointestinal symptoms and fatigue}

GI symptoms were assessed using $100 \mathrm{~mm} \mathrm{VAS}^{(4)}$. Perception of fatigue was evaluated by the Daily Fatigue Impact Scale $(\mathrm{D}-\mathrm{FIS})^{(22)}$ - an eight-item self-report questionnaire, with five options of response per item (from $0=$ no problem, to $4=$ extreme problem). The D-FIS score is derived from the sum of the ordinal scores obtained for each item with possible score range of 0-32. For comparison, VAS ranging from 0 to $100 \mathrm{~mm}$ and with higher values indicating more tiredness was also used ${ }^{(4)}$. Visual analogue scales for GI symptoms and fatigue and D-FIS questionnaire were completed daily during baseline (week 0) while on their habitual diet; during treatment weeks (weeks 1, 4, 7, 10) and during the second wash out week between each treatment (weeks 3, 6, 9) when the volunteers were following their habitual diet (see Fig. 2).

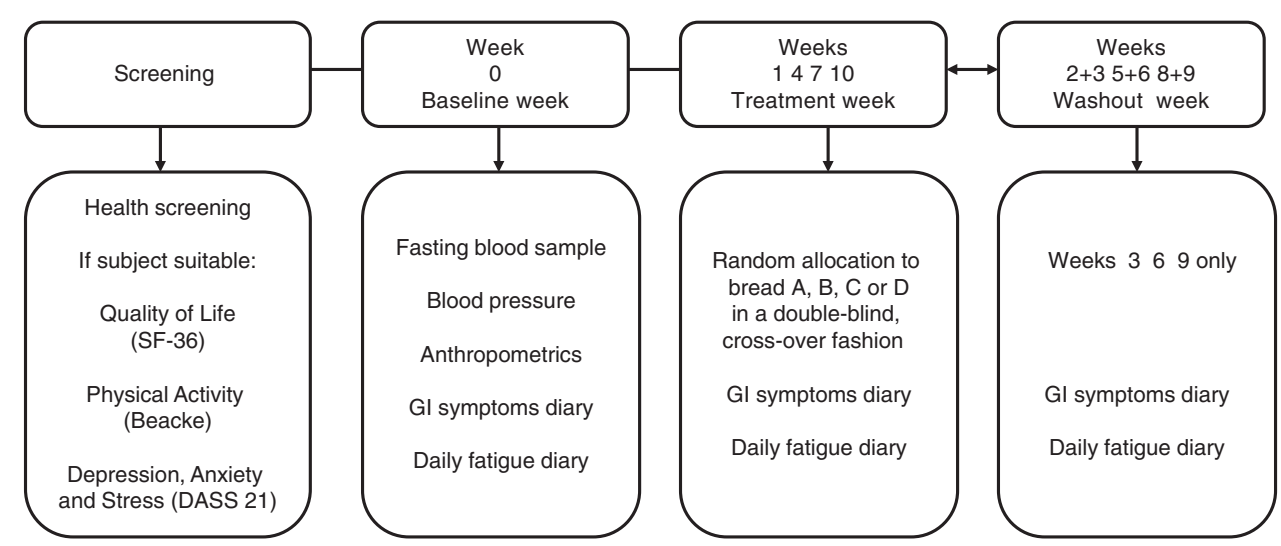

Fig. 2. Study design. After 1 week of a baseline period consuming their habitual diet (week 0 ), subjects were randomly allocated in a double-blind, cross-over fashion to the four breads, labelled as bread A, B, C or D. Each experimental period was followed by a 14-d wash-out period before crossing over to the next bread. SF-36, 36-Item Short Form Health Survey, DASS 21, Depression, Anxiety and Stress Scale; Gl, gastrointestinal. 


\section{Serum analysis}

IgA levels in serum were measured using the Konelab analyser. IgA was purchased from Thermo Fisher Scientific. The detection limit was $0.2 \mathrm{~g} / \mathrm{l}$. The concentrations of anti-human-tissuetransglutaminase IgA (anti-htTG IgA) (Immundiagnostik AG), gliadin IgA and IgG (Genesis Diagnostics Ltd) and zonulin (Immundiagnostik AG) in the serum were measured using specific ELISA. Samples classed as negative were as follows: $<22 \mathrm{AU} / \mathrm{ml}$ (htTG IgA); <4U/ml (gliadin IgA); $<10 \mathrm{U} / \mathrm{ml}$ (gliadin $\mathrm{IgG}$ ); normal reference range for zonulin was $34 \mathrm{ng} / \mathrm{ml}$ $( \pm 14 \mathrm{ng} / \mathrm{ml})$ (data supplied by the manufacturer of the kit).

\section{Whole blood analysis}

The full blood count was determined with a SYSMEX (Eightcheck-3WP Assay Sysmex Europe GmbH) on EDTA treated blood. Measurements were performed within $30 \mathrm{~min}$ of collection, according to the manufacturer's instructions.

Lipid and blood glucose analyses were determined using an Alere Cholestech LDX (LDX Lipid Profile + Glucose cassette supplied by Alere Ltd) on heparin treated blood. Measurements were performed within $30 \mathrm{~min}$ of collection, according to the manufacturer's instructions.

The determination of basophil activation upon wheat allergen stimulation (Flow2 CAST test) was carried out using whole EDTA anti-coagulated blood. Flow2 CAST and wheat allergen were purchased from BÜHLMANN Laboratories AG. Analysis were performed according to the manufacturer's instructions on the day of blood collection, using a Becton-Dickinson FACSCalibur flow cytometer.

\section{Statistical analysis}

Power calculations. The study aimed to detect a minimum shift of 10 VAS units (out of a total of 100 units) in GI scores due to the consumption of the treatment breads, within each of the control and GS groups. Assuming a within volunteer spread of 10 VAS units, a power of $90 \%$ and $5 \%$ significance, this required twelve volunteers (per group). To allow for dropout and non-compliance this was raised to sixteen volunteers for each group.

Assessment of differences in baseline characteristics between control and gluten-sensitive groups. Baseline measurements of the control and GS groups were compared by independent-samples $t$ test. In addition, differences in baseline blood measurements between the two groups were also compared by analysis of variance with covariate adjustment for age and BMI.

Assessment of suitability of Aspergillus niger prolyl endoprotease treated bread for consumption by glutensensitive volunteers, based on gastrointestinal and daily fatigue scores. First, the scores from the habitual diet (obtained during week 0 and the 2 nd week of each washout period, weeks 3, 6 and 9) were analysed to investigate trends over time, based on analysis of variance with volunteer as random effect and week, group and their interaction as fixed effects, followed by post hoc $t$ test comparisons. This indicated that for several GI symptoms the scores were significantly $(P<0.05)$ higher at week 0 and declined over time. This is a well-known psychological effect when participants are entering into a trial ${ }^{(23)}$. Second, the GI and daily fatigue scores obtained during the treatment weeks (weeks 1, 4, 7 and 10) were analysed by ANOVA with volunteer as random effect and with treatment, group and their interaction as fixed effects, and with the corresponding habitual diet score from the preceding washout week as covariate. Third, as comparison of the treatment diets against the habitual diet was of specific interest, an alternative analysis was also performed, where the scores of the habitual diet (weeks 0, 3, 6 and 9) were averaged and, together with the four treatments, analysed as ANOVA with volunteer as random effect and with treatment (including the habitual diet), group and their interaction as fixed effects. The overall findings of this third analysis were similar to those from the covariate analysis (second analysis), and therefore findings from the latter are presented. This was followed by post hoc $t$ tests to compare the scores obtained from the four treatment breads against those of the habitual diet for both control and GS groups.

For all statistical analyses, visual inspection of the residual plots indicated that assumptions of normality and constant variance had been met and therefore no transformations were needed. A $P$ value $<0.05$ indicated statistical significance. Data are presented as means with their standard errors. All statistical analyses were conducted in Genstat 17th Edition (Release 17.1; VSN International Ltd).

\section{Results \\ Subjects}

The baseline characteristics of the subjects in both treatment groups are shown in Table 1. Data are only reported for those subjects who completed the study. There were no significant differences in the baseline characteristics between the two groups with respect to age, BMI, systolic and diastolic blood pressure and pulse.

\section{Analytical results of the breads}

Typically $80 \%$ of the protein in bread is gluten ${ }^{(24)}$. In our study, the control bread contained $7 \cdot 88 \%$ protein, whereas the bread

Table 1. Baseline characteristics of the control (CON) and gluten-sensitive (GS) treatment groups

(Mean values with their standard errors)

\begin{tabular}{|c|c|c|c|c|c|}
\hline & \multicolumn{2}{|c|}{ CON group } & \multicolumn{2}{|c|}{ GS group } & \multirow[b]{2}{*}{$P^{\star}$} \\
\hline & Mean & SEM & Mean & SEM & \\
\hline No. of subjects & \multicolumn{2}{|c|}{12} & \multicolumn{2}{|c|}{16} & \\
\hline Age (years) & $58 \cdot 8$ & $2 \cdot 9$ & $51 \cdot 1$ & 3.6 & 0.12 \\
\hline BMI $\left(\mathrm{kg} / \mathrm{m}^{2}\right)$ & $26 \cdot 0$ & 1.3 & 23.6 & 0.7 & 0.081 \\
\hline Systolic blood pressure (mmHg) & 135.5 & $7 \cdot 2$ & $124 \cdot 2$ & 4.7 & $0 \cdot 18$ \\
\hline Diastolic blood pressure $(\mathrm{mmHg})$ & 74.5 & 3.1 & $72 \cdot 8$ & $2 \cdot 7$ & 0.69 \\
\hline Pulse (heartbeats/min) & $64 \cdot 2$ & 1.8 & 61.9 & $2 \cdot 1$ & 0.43 \\
\hline
\end{tabular}

${ }^{*}$ Two-sample $t$ test. 
Table 2. Analysis of the experimental breads (Mean values with their standard errors; $n$ 2)

\begin{tabular}{lcccccc}
\hline & \multicolumn{2}{c}{$\begin{array}{c}\text { Protein } \\
(\%)\end{array}$} & & \multicolumn{2}{c}{$\begin{array}{c}\text { Moisture } \\
(\%)\end{array}$} & \\
\cline { 2 - 3 } Types of bread & Mean & SEM & & Mean & SEM & Protein dmb (\%) \\
\cline { 2 - 3 } Control bread & 7.88 & 0.01 & & 43.1 & 0.2 & 13.8 \\
Biscuit flour bread & 6.63 & 0.01 & & 38.1 & 0.2 & 10.7 \\
0.8\% ANPEP (340 PPU/kg) & 8.64 & 0.01 & & 39.2 & 0.1 & 14.2 \\
$1 \%$ ANPEP (430 PPU/kg) & 8.65 & 0.01 & 39.1 & 0.1 & 14.2 \\
\hline
\end{tabular}

$\mathrm{dmb}$, DM base; ANPEP, Aspergillus niger prolyl endoprotease; PPU, propyl peptidase units.

Table 3. Gluten content of the bread* (Mean values with their standard errors; $n$ 3)

\begin{tabular}{|c|c|c|c|c|}
\hline \multirow[b]{3}{*}{ Types of bread } & \multicolumn{2}{|c|}{$a-20$ gliadin } & \multicolumn{2}{|c|}{ R5 epitope } \\
\hline & \multicolumn{2}{|c|}{ Gluten - Rel. (\%) } & \multicolumn{2}{|c|}{ Gluten - Rel. (\%) } \\
\hline & Mean & SEM & Mean & SEM \\
\hline Control bread & $100 \cdot 0^{a}$ & 2.5 & $100 \cdot 0^{a}$ & 4.4 \\
\hline Biscuit flour bread & $88 \cdot 1^{\mathrm{a}}$ & 4.9 & $83 \cdot 6^{\mathrm{b}}$ & $1 \cdot 2$ \\
\hline $0.8 \%$ ANPEP & $58 \cdot 4^{b}$ & 2.7 & $57 \cdot 1^{\mathrm{C}}$ & 3.6 \\
\hline $1 \%$ ANPEP & $61 \cdot 5^{b}$ & $1 \cdot 1$ & $59 \cdot 0^{\mathrm{c}}$ & 2.9 \\
\hline
\end{tabular}

ANPEP, Aspergillus niger prolyl endoprotease.

a,b,c Mean values in the same column with unlike superscript letters are significantly different - post hoc comparisons $(P<0.001)$.

* Data are normalised to the control bread (100\%). Data were analysed by oneway ANOVA.

prepared with biscuit flour was approximately $16 \%$ lower in protein than the control bread. As a consequence of the addition of the enzyme, both ANPEP treated breads contained more protein in relation to the control bread (Table 2).

The level of gluten degradation was evaluated by two different ELISA assays, one sensitive to the $\alpha$-20 gliadin, the other to the R5 epitope. Both assays showed that ANPEP treated breads contained approximately $40 \%$ less immunoreactive gluten than the control bread. However, increasing the concentrations of ANPEP from $0.8 \% \mathrm{w} / \mathrm{w}$ to $1.0 \% \mathrm{w} / \mathrm{w}$ did not increase the degradation of the gluten. The gluten content of bread prepared with biscuit flour was approximately $16 \%$ lower than that of the control bread and consistent with its lower protein content (Table 3).

\section{Lifestyles in relation to gluten consumption}

During the process of recruitment the subjects' experiences, personal histories and understanding of gluten were assessed using questionnaires and an interview (sixty-two subjects were interviewed, twelve of these were healthy controls). Of the fifty GS volunteers with no diagnosis of coeliac disease, that completed the questionnaires, eleven (22\%) followed a strict GFD with the remaining thirty-nine subjects (78\%) following a lowgluten lifestyle by mainly substituting wheat bread and pasta with GF options or removing those items from their diet altogether. With the exception of two subjects, those who were following a strict GFD declined to participate. Those individuals, who consented to take part, were mostly GS

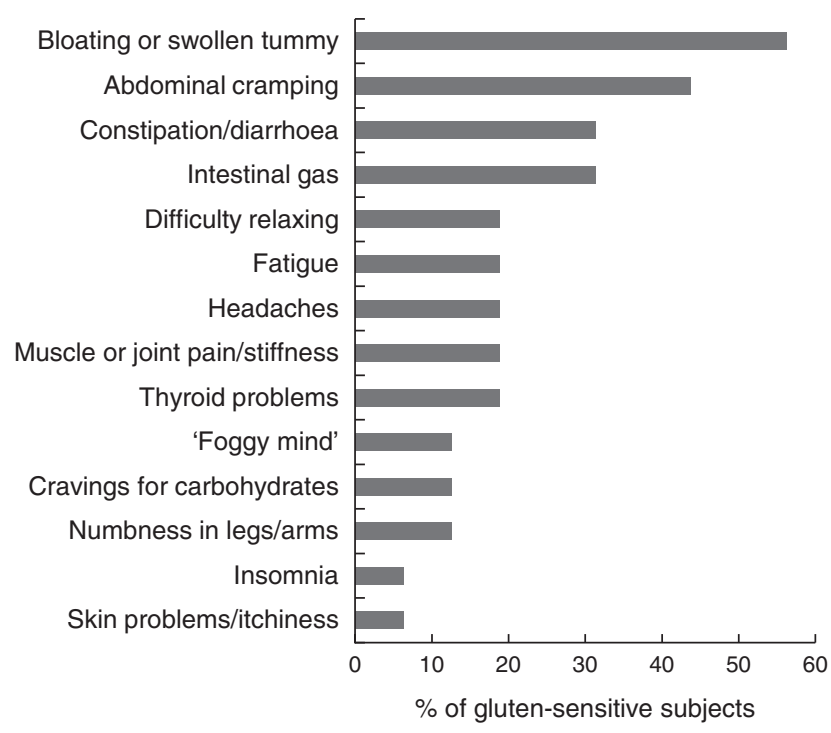

Fig. 3. Frequency of gastrointestinal and extra-intestinal symptoms reported by the subjects in the gluten-sensitive group before adopting a low-gluten lifestyle.

subjects with a low-gluten lifestyle, whose habitual diet still contained variable quantities of gluten. Fig. 3 shows the range and frequency of GI and extra-intestinal manifestations (selfperceived as gluten-related), reported by the GS study group before adopting a low-gluten lifestyle.

\section{Biomarkers for coeliac disease and wheat allergy}

The total IgA levels were within the normal range in all participants, between 0.7 to $3.6 \mathrm{~g} / \mathrm{l}$. For all the GS participants the IgA anti-tTG values for CD-associated antibodies were lower than the accepted levels for diagnosis of $\mathrm{CD}(<22 \mathrm{AU} / \mathrm{ml})$. Gliadin IgA was weakly positive (value $5 \mathrm{U} / \mathrm{ml} \mathrm{IgA}$ ) for one participant in the GS group (cut-off values indicated by the manufacturer $>4 \mathrm{U} / \mathrm{ml}$ IgA), the levels for all other samples in the GS and control groups were negative. A positive result for Gliadin IgG was observed in $18 \%$ of the GS participants who had values $>10 \mathrm{U} / \mathrm{ml}$ IgG. There was also one positive sample in the control group. Serum zonulin levels were in the $(34$ (SEM 14) $\mathrm{ng} / \mathrm{ml}$ ) range (as per manufacturer's data sheet) for all participants in both groups, with the exception of one control sample. No GS subjects with a wheat allergy were identified upon basophil activation with a wheat allergen (data not shown).

\section{Whole blood results}

The GS subjects had 9\% lower erythrocyte concentration $(P=0.02), 6 \%$ higher mean corpuscular volume (MCV) $(P=0.002), 4 \%$ higher mean corpuscular $\mathrm{Hb}(P=0.03), 13 \%$ higher platelet distribution width (PDW) $(P=0.01)$ and $10.5 \%$ higher mean platelet volume (MPV) $(P=0.005)$ in comparison with the control group (independent-samples $t$ tests). The values remained significantly different after adjusting for age and BMI as covariates (Table 4).

The GS group had 18\% lower total cholesterol levels than the control group (independent-samples $t$ test; $P=0.018$ ). 
However, this was not significant after adjustment for age and BMI ( $P=0 \cdot 082)$. Subjects in the GS group had significantly higher HDL values in comparison with the control group (independentsamples $t$ test; 1.4 (SEM 0.1) $v .1 .7$ (SEM 0.1); $P=0.021$ ). After adjustment for age and BMI, differences in HDL were no longer significant. GS subjects had $30 \%$ lower LDL levels $(P=0.005)$ and the LDL:HDL ratio was 3.2 (SEM 0.4) in the control $v$. 1.8 (SEM $0 \cdot 2$ ) in the GS group $(P=0.003)$. These remained significant after adjustment for age and BMI (for LDL $P=0 \cdot 008$; for LDL:HDL ratio $P=0.005$ ) (Table 4). There were no differences in the blood glucose concentrations between the two groups (Table 4).

\section{Gastrointestinal symptoms}

There were no differences in the severity of the GI symptoms measured $(P>0.095)$ between the control and GS group while on their habitual diet (Table 5). There were no significant effects of dietary treatment, and there were no significant interactions with participant group. There were however differences between the two groups after consumption of the experimental breads. The scores for bloating (15 VAS units; $P=0 \cdot 008$ ), flatulence (13 VAS units; $P=0 \cdot 003$ ), pain (8 VAS units; $P=0.045$ ) and rumbles ( 8 VAS units; $P=0.04$ )

Table 4. Comparison of full blood count (FBC), lipid profile and blood glucose measurements between control (CON) and glutensensitive (GS) group at baseline

(Mean values with their standard errors)

\begin{tabular}{|c|c|c|c|c|c|c|}
\hline \multirow[b]{2}{*}{ FBC, lipid and blood glucose parameters } & \multirow[b]{2}{*}{ Unit } & \multicolumn{2}{|c|}{ CON group } & \multicolumn{2}{|c|}{ GS group } & \multirow[b]{2}{*}{$P^{*}$} \\
\hline & & Mean & SEM & Mean & SEM & \\
\hline Leucocyte concentration & $\times 10^{3} / \mu \mathrm{l}$ & $5 \cdot 0$ & 0.4 & $5 \cdot 0$ & 0.3 & NS \\
\hline Erythrocyte concentration & $\times 10^{6} / \mu \mathrm{l}$ & 4.6 & 0.1 & $4 \cdot 2$ & 0.1 & 0.022 \\
\hline $\mathrm{Hb}$ concentration & $\mathrm{mmol} / \mathrm{l}$ & 2.09 & 0.02 & 2.03 & 0.05 & NS \\
\hline Haematocrit & $\%$ & $40 \cdot 1$ & 0.4 & 39.1 & 0.8 & NS \\
\hline Mean corpuscular volume & $\mathrm{fl}$ & $88 \cdot 1$ & 1.0 & 92.4 & $0 \cdot 8$ & 0.002 \\
\hline Mean corpuscular $\mathrm{Hb}$ & $\mathrm{pg}$ & 29.8 & 0.3 & $31 \cdot 1$ & 0.4 & 0.022 \\
\hline Mean cell (erythrocyte) $\mathrm{Hb}$ concentration & $\mathrm{mmol} / \mathrm{l}$ & $5 \cdot 24$ & 0.02 & $5 \cdot 21$ & 0.03 & NS \\
\hline Platelet concentration & $\times 10^{3} / \mu \mathrm{l}$ & $230 \cdot 6$ & 13.7 & $212 \cdot 3$ & 11.6 & NS \\
\hline Percentage of lymphocytes & $\%$ & $33 \cdot 2$ & 3.0 & $34 \cdot 3$ & 1.9 & NS \\
\hline Percentage of basophils, eosinophils and monocytes & $\%$ & $9 \cdot 7$ & 0.9 & $8 \cdot 7$ & 0.5 & NS \\
\hline Percentage of neutrophils & $\%$ & $57 \cdot 2$ & $3 \cdot 2$ & $57 \cdot 0$ & $2 \cdot 1$ & NS \\
\hline Lymphocyte concentration & $\times 10^{3} / \mu l$ & 1.6 & 0.1 & 1.6 & 0.1 & NS \\
\hline Basophil, eosinophil and monocyte concentration & $\times 10^{3} / \mu \mathrm{l}$ & 0.5 & 0.0 & 0.4 & 0.0 & NS \\
\hline Neutrophil concentration & $\times 10^{3} / \mu \mathrm{l}$ & 3.0 & 0.4 & $2 \cdot 9$ & 0.3 & NS \\
\hline Erythrocyte distribution width-SD & $\mathrm{fl}$ & $44 \cdot 3$ & $0 \cdot 8$ & $46 \cdot 5$ & $0 \cdot 6$ & 0.012 \\
\hline Erythrocyte distribution width-CV & $\%$ & $12 \cdot 4$ & 0.2 & $12 \cdot 4$ & 0.2 & NS \\
\hline Platelet distribution width & $\mathrm{fl}$ & $10 \cdot 7$ & $0 \cdot 2$ & $12 \cdot 1$ & 0.4 & 0.027 \\
\hline Mean platelet volume & $\mathrm{fl}$ & 8.5 & 0.1 & $9 \cdot 4$ & 0.2 & 0.020 \\
\hline Plateletcrit & $\%$ & 0.2 & 0.0 & 0.2 & 0.0 & NS \\
\hline Total cholesterol & $\mathrm{mmol} / \mathrm{l}$ & $5 \cdot 8$ & 0.3 & $4 \cdot 8$ & 0.2 & 0.082 \\
\hline TAG & $\mathrm{mmol} / \mathrm{l}$ & $1 \cdot 2$ & 0.2 & 0.9 & 0.1 & NS \\
\hline HDL & $\mathrm{mmol} / \mathrm{l}$ & 1.4 & $0 \cdot 1$ & $1 \cdot 7$ & $0 \cdot 1$ & NS \\
\hline LDL & $\mathrm{mmol} / \mathrm{l}$ & 4.0 & 0.3 & $2 \cdot 8$ & 0.2 & 0.008 \\
\hline Glucose & $\mathrm{mmol} / \mathrm{l}$ & 4.9 & 0.2 & 4.9 & 0.1 & NS \\
\hline LDL:HDL & & $3 \cdot 2$ & 0.4 & $1 \cdot 8$ & 0.2 & 0.005 \\
\hline
\end{tabular}

* From ANOVA with age and BMI as covariates. Control group ( $n$ 12), GS group ( $n$ 16).

Table 5. Gastrointestinal (GI) symptoms experience in control (CON) and gluten-sensitive (GS) groups before and after consumption of different treatment breads $†$ (Mean values with their standard errors)

\begin{tabular}{|c|c|c|c|c|c|c|c|c|c|c|c|c|c|c|c|c|c|c|c|c|}
\hline \multirow[b]{3}{*}{ Gl symptoms } & \multicolumn{4}{|c|}{ Habitual diet } & \multicolumn{4}{|c|}{ Control bread } & \multicolumn{4}{|c|}{ Biscuit flour bread } & \multicolumn{4}{|c|}{$0.8 \%$ ANPEP bread } & \multicolumn{4}{|c|}{$1 \%$ ANPEP bread } \\
\hline & \multicolumn{2}{|c|}{ CON } & \multicolumn{2}{|c|}{ GS } & \multicolumn{2}{|c|}{$\mathrm{CON}$} & \multicolumn{2}{|c|}{ GS } & \multicolumn{2}{|c|}{ CON } & \multicolumn{2}{|c|}{ GS } & \multicolumn{2}{|c|}{$\mathrm{CON}$} & \multicolumn{2}{|c|}{ GS } & \multicolumn{2}{|c|}{$\mathrm{CON}$} & \multicolumn{2}{|c|}{ GS } \\
\hline & Mean & SEM & Mean & SEM & Mean & SEM & Mean & SEM & Mean & SEM & Mean & SEM & Mean & SEM & Mean & SEM & Mean & SEM & Mean & SEM \\
\hline مa & $5 \cdot 1$ & $2 \cdot 7$ & 6.0 & 1.9 & $2 \cdot 7$ & 0.9 & $6 \cdot 7$ & 1.9 & $4 \cdot 2$ & 1.3 & $8 \cdot 1$ & $2 \cdot 2$ & $5 \cdot 6$ & $3 \cdot 1$ & 8.9 & 2.5 & $5 \cdot 4$ & $2 \cdot 4$ & 8.4 & $2 \cdot 1$ \\
\hline Bloating & 6.9 & $2 \cdot 8$ & $15 \cdot 5$ & 4.0 & $5 \cdot 0$ & 1.5 & $19 \cdot 0$ & 3.5 & $5 \cdot 8$ & $2 \cdot 1$ & $19 \cdot 1$ & $4 \cdot 3$ & $5 \cdot 8$ & $2 \cdot 9$ & $20 \cdot 9$ & $5 \cdot 1$ & $6 \cdot 1$ & $2 \cdot 2$ & $20 \cdot 0$ & 3.4 \\
\hline Flatulence & $11 \cdot 0$ & 2.9 & $19 \cdot 6$ & 3.4 & $9 \cdot 2$ & $2 \cdot 2$ & $24 \cdot 3$ & 3.4 & $11 \cdot 1$ & $2 \cdot 8$ & $28 \cdot 6^{\star \star}$ & 3.8 & $12 \cdot 4$ & $4 \cdot 1$ & $22 \cdot 3$ & $4 \cdot 7$ & 11.0 & 2.5 & $24 \cdot 6$ & $3 \cdot 3$ \\
\hline Pain & $6 \cdot 9$ & $2 \cdot 9$ & $9 \cdot 6$ & $2 \cdot 5$ & 4.5 & 1.7 & $16 \cdot 3^{\star \star}$ & $3 \cdot 2$ & $5 \cdot 0$ & $2 \cdot 0$ & $12 \cdot 0$ & $2 \cdot 7$ & $6 \cdot 1$ & $2 \cdot 9$ & 11.5 & $3 \cdot 6$ & $5 \cdot 6$ & $2 \cdot 1$ & $12 \cdot 4$ & $2 \cdot 5$ \\
\hline Rumbles & 7.9 & $3 \cdot 1$ & $12 \cdot 4$ & $2 \cdot 8$ & $6 \cdot 2$ & 2.5 & $17 \cdot 5^{\star \star}$ & 3.2 & $7 \cdot 2$ & $2 \cdot 3$ & $17 \cdot 2^{*}$ & $3 \cdot 3$ & 8.9 & $3 \cdot 2$ & $13 \cdot 3$ & $3 \cdot 2$ & $7 \cdot 6$ & $3 \cdot 1$ & $16 \cdot 2$ & $2 \cdot 5$ \\
\hline
\end{tabular}

ANPEP, Aspergillus niger prolyl endoprotease.

Significance of comparison against the habitual diet within group: * $P<0.05,{ }^{* *} P<0.01$.

† Control group ( $n$ 12), GS group ( $n$ 16). Data were analysed by ANOVA with random effect for volunteer and fixed effects for diet, group and its interaction. The effect of group was significant for bloating, flatulence, pain and rumbles. There were no significant effects of diet or its interaction with group. Comparison symptom scores of the various test breads against those observed during the habitual diet was of specific interest and therefore post hoc $t$ test was performed to compare, within each group, Gl symptoms during the treatment breads against symptoms when following the habitual diet. 
were higher in the GS group when compared with the control (Fig. 4).

Of specific interest was the comparison of GI symptoms while on the experimental breads against the symptoms observed when following the habitual diet. So although there was no overall significant effect of treatment, post hoc testing was performed to compare, within each group, the experimental bread against the habitual diet. When comparing the breads against habitual diet within the control group, none of the experimental breads had any significant effects on the GI symptoms measured. For the GS group however, consumption of all experimental breads numerically increased GI symptoms severity in comparison with the normal habitual diet (Table 5). These increases reached statistical significance for some GI symptoms only with control bread (pain, 10 VAS units; $P=0.007$; rumbles, 11 VAS units; $P=0 \cdot 040)$ and bread made with biscuit flour (flatulence, 21 VAS units; $P=0.006$ ), with rumbles showing a tendency to increase (rumbles, 9 VAS units; $P=0.052$ ) (Table 5).

\section{Extra-intestinal symptoms}

The comparison of the fatigue scores (using D-FIS) in the group of healthy individuals $v$. the GS group, showed numerically

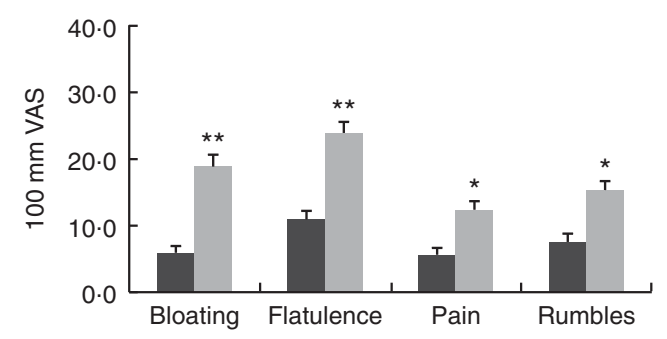

Fig. 4. Gastrointestinal symptoms experience in the control and glutensensitive groups. Values are means, with their standard errors over all five treatments combined (habitual diet and four treatment breads). Control group ( $n$ 12, $\square$ ), gluten-sensitive (GS, $\square$ ) group ( $n$ 16). Data were analysed by ANOVA with random effect for participants and fixed effects for group, treatment and their interaction. Significant effects for group: ${ }^{*} P<0.05$, ${ }^{* *} P<0.01$. There were no significant effects for treatment and its interaction with group. VAS, visual analogue scale. better scores in the healthy controls (e.g. lower level of fatigue) compared with the GS group, but this did not reach statistical significance $(P>0.05)$ (Table 6$)$. The same pattern was observed with the scoring on the fatigue VAS scale.

The Baecke questionnaire was used for the evaluation of habitual physical activity. It consists of sixteen questions and separates the person's physical activity into three categories: physical activity during work, sports and leisure time. The GS group had a statistically significant higher leisure index compared with the control group $(2 \cdot 6 v \cdot 3 \cdot 3 ; P=0 \cdot 006)$ (Table 7).

SF-36 survey for measurement of health status yields an eight-subscale profile of functional health and well-being. Comparison of the SF-36 scores did not reveal any statistically significant differences between the control and GS group with respect to any of the SF-36 subscales (Table 7).

Table 7. Comparison of the indices of physical activity, 36-Item Short Form Health Survey (SF-36) scale and Depression, Anxiety and Stress Scale (DASS-21) subscales between control (CON, $n$ 12) and glutensensitive (GS, $n$ 16) group at baseline

(Mean values with their standard errors)

\begin{tabular}{|c|c|c|c|c|c|}
\hline \multirow[b]{2}{*}{ Physical activity (Baecke) } & \multicolumn{2}{|c|}{ CON group } & \multicolumn{2}{|c|}{ GS group } & \multirow[b]{2}{*}{$P^{\star}$} \\
\hline & Mean & SEM & Mean & SEM & \\
\hline Work index & $2 \cdot 4$ & 0.2 & 2.5 & 0.1 & 0.77 \\
\hline Sport index & $2 \cdot 2$ & 0.2 & $2 \cdot 7$ & 0.2 & 0.075 \\
\hline Leisure index & 2.6 & 0.2 & 3.3 & 0.1 & 0.006 \\
\hline \multicolumn{6}{|l|}{ SF-36 subscales } \\
\hline Physical functioning & $86 \cdot 7$ & 3.7 & 93.8 & 1.7 & 0.1 \\
\hline Role - physical & $91 \cdot 7$ & $8 \cdot 3$ & $78 \cdot 1$ & $10 \cdot 2$ & 0.34 \\
\hline Bodily pain & $87 \cdot 7$ & 4.4 & $75 \cdot 8$ & 5.4 & 0.12 \\
\hline General health & $80 \cdot 1$ & 3.9 & $81 \cdot 1$ & 4.2 & 0.87 \\
\hline Vitality & 67.5 & $5 \cdot 0$ & $63 \cdot 1$ & 5.6 & 0.58 \\
\hline Social functioning & 94.8 & $2 \cdot 9$ & $89 \cdot 1$ & 4.8 & 0.36 \\
\hline Role - emotional & $91 \cdot 7$ & $8 \cdot 3$ & $91 \cdot 7$ & 5.7 & 1 \\
\hline Mental health & $80 \cdot 7$ & 3.3 & $75 \cdot 8$ & 3.9 & 0.36 \\
\hline \multicolumn{6}{|l|}{ DASS-21 subscales } \\
\hline Stress & 9.2 & 2.5 & $9 \cdot 1$ & 1.9 & 0.97 \\
\hline Anxiety & 4.2 & 1.3 & $4 \cdot 2$ & 1.0 & 0.98 \\
\hline Depression & 3.4 & 1.2 & $5 \cdot 1$ & 1.9 & 0.5 \\
\hline
\end{tabular}

* One-way ANOVA adjusted for age and BMI.

Table 6. Comparison of fatigue experience between control (CON) and gluten-sensitive (GS) groups before and after consumption of different treatment breads (Mean values with their standard errors)

\begin{tabular}{|c|c|c|c|c|c|c|c|c|c|c|c|c|c|}
\hline \multirow[b]{3}{*}{ Gl symptoms } & \multicolumn{10}{|c|}{ Type of bread* } & & & \\
\hline & \multicolumn{2}{|c|}{ Habitual diet } & \multicolumn{2}{|c|}{ Control } & \multicolumn{2}{|c|}{ Biscuit flour } & \multicolumn{2}{|c|}{$0.8 \%$ ANPEP } & \multicolumn{2}{|c|}{$1 \%$ ANPEP } & \multicolumn{3}{|c|}{$P \dagger$} \\
\hline & Mean & SEM & Mean & SEM & Mean & SEM & Mean & SEM & Mean & SEM & grp & treat & grp $\times$ treat \\
\hline Control group (D-FIS) & $2 \cdot 9$ & 1.4 & $2 \cdot 4$ & 1.3 & 1.6 & 0.8 & $2 \cdot 3$ & $1 \cdot 1$ & $2 \cdot 1$ & 0.9 & & & \\
\hline Gluten-sensitive group (D-FIS) & $5 \cdot 1$ & $1 \cdot 8$ & $6 \cdot 4$ & 1.8 & $4 \cdot 4$ & $1 \cdot 3$ & $6 \cdot 1$ & $2 \cdot 0$ & $5 \cdot 2$ & $1 \cdot 6$ & & & \\
\hline Control group (VAS) & 13.5 & $5 \cdot 7$ & $11 \cdot 1$ & $5 \cdot 2$ & $10 \cdot 0$ & 4.5 & 14.4 & 5.9 & $12 \cdot 3$ & $4 \cdot 8$ & 0.14 & 0.16 & 0.71 \\
\hline Gluten-sensitive group (VAS) & $19 \cdot 3$ & $4 \cdot 8$ & 24.5 & $5 \cdot 2$ & $19 \cdot 2$ & 3.8 & $23 \cdot 8$ & $5 \cdot 3$ & $16 \cdot 4$ & $3 \cdot 7$ & 0.21 & 0.20 & 0.37 \\
\hline
\end{tabular}

ANPEP, Aspergillus niger prolyl endoprotease; grp, group; treat, treatment; D-FIS, Daily Fatigue Impact Scale; VAS, visual analogue scale.

* $n 12$ for control group; $n 16$ for GS group.

† Data were analysed by ANOVA with random effect for participants and fixed effects for grp, treat and their interaction. 
The Depression, Anxiety and Stress Scale is a twenty-oneitem self-report scale designed to measure the emotional states of depression, anxiety and stress. There were no statistically significant differences between the control and GS groups with respect to the three DASS-21 subscales (Table 7).

\section{Discussion}

This study shows that individuals who are self-defined as being sensitive to gluten differ from healthy controls in several measurable ways and show an increase in the severity of GI symptoms when challenged with normal bread.

Two distinct groups of GS subjects were identified: those who follow a strict GFD (22\% of those who responded) and those who follow a low-gluten lifestyle by removing 'normal' bread and pasta (and occasionally other gluten containing items) from their diets or substituting them with GF options as a relatively easy way of avoiding excess gluten. The attitude to a strict GFD depends on personal experience; individuals with severe symptoms are committed to a GFD because of the impact it has on their health, whereas those with mild discomfort focus on following a low-gluten lifestyle. The qualitative data from both, the questionnaire and from interviews with the volunteers, suggest that whilst this latter group self-define themselves as being sensitive to gluten, they still have a variable but reduced gluten intake and are not gluten-free.

Bread-making wheat typically contains ca $11 \%$ protein, $80 \%$ of which is gluten. This equates to an intake of approximately $9 \mathrm{~g}$ of gluten/ $100 \mathrm{~g}$ of bread $^{(24)}$. Consumption of bread in the UK is estimated to be approximately $90 \mathrm{~g} / \mathrm{d}^{(25,26)}$, thus average adult consumption of gluten in bread is approximately $8 \mathrm{~g} / \mathrm{d}$. Three slices of the control bread provided the volunteers with $6.6 \mathrm{~g}$ of gluten/d. The biscuit flour bread provided the volunteers with around $5.6 \mathrm{~g}$ gluten/d. Treatment of the flour with ANPEP reduced the amount of gluten by approximately $40 \%$ and provided the volunteers with $2.6 \mathrm{~g}$ of gluten/d. It is unclear why there was no additional reduction of the gluten content in bread treated with $1 \%$ ANPEP (as was anticipated) when compared with 0.8\% ANPEP.

There were no differences in the severity of the GI symptoms measured between the control and GS groups while on their habitual diet, suggesting that following a low-gluten lifestyle reduces the symptoms to tolerable levels in those GS sufferers with mild complaints.

The absence of GI symptoms in the control group suggest that the proline-specific ANPEP is well tolerated and can be used in the production of bread which retains taste, texture and appearance despite the $40 \%$ reduction of immunoreactive gluten. In contrast, the individuals in the GS group experienced more noticeable GI symptoms while consuming the experimental breads. When the data were combined (Fig. 4), the measured GI symptoms in the control group were within the physiological range ( $<10 \mathrm{~mm}$ VAS), effectively no symptoms, whereas the GS group reported mild GI discomfort (up to $30 \mathrm{~mm}$ VAS). Indeed, the majority of GS subjects, who agreed to participate in the trial, reported during the screening process that they experienced mild symptoms. Furthermore, two of the participants who reported more severe symptoms (and were on a strict GFD) dropped out during the 1st treatment week when consuming either control bread or bread made with biscuit flour. Therefore, it is possible that an unintended sample bias has been introduced as the true effect is underestimated by excluding volunteers in the higher scoring range. This may be an important weakness in this and similar studies and should be considered in future designs.

All of the experimental breads numerically increased the severity of the GI symptoms experienced by the GS group in comparison with their habitual diet (Table 5). These increases reached significance for some GI symptoms only with the control and biscuit flour bread. Although the ANPEP bread failed to reach statistical difference for increasing symptoms in comparison with the subjects' habitual diet, the overall magnitude of the effects were similar for all four treatments. This may be due to levels of gluten in the ANPEP bread being insufficiently reduced. Also, the absence of a treatment effect needs to be considered in the light of the difficulties encountered working with this group of subjects. A nocebo effect, a strong anticipatory symptomatic response, is often reported in NCGS populations $^{(8,27)}$ and there is a possibility that the positive symptomatic responses observed across all treatment arms are a result of a nocebo effect. A placebo, gluten-free treatment, was not included in this trial as it is virtually impossible to produce gluten-free bread, with similar qualities of taste and texture to bread prepared with wheat flour. As volunteers would have been readily able to distinguish the gluten-free product, a placebo would have been rendered useless.

The investigation into the exclusion of coeliac disease and wheat allergy was initially through health screening and gluten sensitivity questionnaires and an interview. There was considerable variation in the pathway and time of the diagnostic tests for the previous exclusion of coeliac disease and wheat allergy. Most of the participants had not had a duodenal biopsy as their GP ruled out the diagnosis based on negative serology tests. These tests took place between 5 months and 18 years before the start of the study.

The National Institute for Health and Care Excellence guideline published in September 2015 states that any test for $\mathrm{CD}$ is accurate only if a gluten-containing diet is eaten during the diagnostic process and that some gluten has to be eaten in more than one meal every day for at least 6 weeks before testing ${ }^{(28)}$. IgA tissue transglutaminase (IgA tTG) is most commonly used as the first test to detect the presence of coeliac disease antibodies. This test should be done after testing for IgA deficiency. IgA deficiency is defined as total IgA $<0.07 \mathrm{~g} / \mathrm{l}$ and will result in a false negative result when tested for IgA antibody ${ }^{(28)}$. As the participants in this study were following a lowgluten diet as opposed to a GFD and considering the variations in their previous experience in testing for $\mathrm{CD}$ we performed inhouse CD diagnosis tests as described above. However, although all GS participants had negative IgA tTG serology, there is still the possibility that the levels of gluten intake in their diet were not sufficient to trigger a high enough titre for detection of tTG antibodies.

Besides the use of the 'anti-self' tTG antibodies, we also performed measurements for 'anti-gluten' antibodies - antigliadin IgG and IgA. These have been previously reported to have high titres in subjects with gluten sensitivity ${ }^{(29)}$ and 
anti-gliadin IgG test was positive in $57 \%$ of the cases in subjects consuming normal gluten-containing diet ${ }^{(29)}$. In our study the anti-gliadin $\mathrm{IgG}$ test was positive in only $18 \%$ of the GS participants and there was one positive sample in the control group. One possible explanation for the differences in our results is that the low-gluten diet did not sufficiently challenge the production of anti-gliadin IgG in some of the participants. Indeed, it is a widely held view that IgG testing can be used to monitor exposure to gluten, but a positive test does not confirm the presence of food intolerance ${ }^{(30)}$.

Zonulin is a type of protein involved in the regulation of intercellular tight junctions of the intestinal cells ${ }^{(31)}$. Gluten is one of the most powerful triggers for the production and release of zonulin $^{(32)}$ and a link between zonulin and diseases such as coeliac disease and dermatitis herpetiformis has already been established $^{(32,33)}$. For this reasons we investigated zonulin levels in self-defined GS subjects on a low-gluten diet and compared the results to the healthy controls. In this study we find no elevated levels of serum zonulin the GS participants.

Our results show a trend for lower total cholesterol level and significantly lower LDL levels $(P=0 \cdot 008)$ and LDL:HDL ratio in the GS group, when compared with the non-GS group (Table 4). Coeliac disease is associated with low cholesterol levels, which is thought to contribute to a favourable cardiovascular risk profile ${ }^{(34,35)}$. Our findings confirm similarities in the lipid profile of gluten-related disorders, suggesting that the possible mechanisms involved in altering this profile may be linked to the ingestion of gluten.

Anaemia can be the only finding in some patients with coeliac disease ${ }^{(36)}$ and is the most frequent extra-intestinal symptom related to the condition ${ }^{(37)}$. However, evidence for diagnosis of $\mathrm{CD}$ can be found in cases without anaemia, but with an unexplained abnormality on a full blood count, such as erythrocyte macrocytosis ${ }^{(38,39)}$. Our results show that although there were no significant differences between the $\mathrm{Hb}$ levels in the two groups, subjects with self-defined sensitivity to gluten had significantly higher MCV and $\mathrm{MCH}$ when compared with the control group (Table 4). MCV (erythrocyte size) together with erythrocyte distribution width (variability in size of the erythrocytes) were used by Bessman et al. ${ }^{(40)}$ to construct an improved classification of anaemias.

Systemic inflammation is found to be behind the micronutrient deficiencies in patients with $\mathrm{CD}$ even in absence of macroscopic damage to the proximal small bowel ${ }^{(41)}$. Our study also demonstrates higher values for MPV and platelet distribution (or size deviation) width (PDW) in GS subjects compared with healthy controls. This might be indicative of platelet destruction, leading to more freshly formed or younger platelets, which are larger in size, circulating in the bloodstream. A possible association between MPV and different inflammatory conditions has been reported in the literature ${ }^{(42,43)}$ and the results in the present study may reflect an on-going intestinal inflammation.

Fatigue is a manifestation reported by subjects experiencing gluten sensitivity ${ }^{(4,27)}$. Information on the impact of fatigue on quality of life and mental status (depression, anxiety and stress) in the GS population is limited. In addition, to the best of our knowledge, no comparisons have previously been made with results obtained in a healthy control group. In our study, the fatigue index for the GS group, although numerically higher, was comparable with that of the control group, for example no clinical implication was confirmed. Moreover, comparisons within the GS group did not reveal increased levels of tiredness during the treatment periods when compared with the normal habitual diet periods. These outcomes are not in line with the results of the study conducted by Biesiekierski et al. ${ }^{(4)}$ which reported prominent effects on tiredness in this population, whereas on a gluten-containing diet. Our study has the advantage of determining the degree of the perception of fatigue in healthy people as a physiological benchmark for comparison and also taking into account other factors such as physical activity.

Compared with the control group, the GS group scored higher on the physical activity for leisure index. This suggests that the GS group makes more health oriented choices for keeping active during their leisure time and this may be partly the reason for the lack of fatigue in the GS group, as it has been shown that physical activity is one of the best ways to handle fatigue ${ }^{(44)}$

Previous studies have shown that short-term exposure to gluten may have a negative effect on the mental state of people who believe that they are sensitive to gluten ${ }^{(45,46)}$. In this study, both the control and the GS group were within the normal range for all three subscales for depression, anxiety and stress and no differences between the two groups were identified. Our results confirm the findings of Brottveit et al. ${ }^{(47)}$ who report no elevated scores for anxiety and depression.

The SF-36 survey is useful in the assessment of the quality of life in relation to health. In this study comparison of the SF-36 scores did not reveal any differences between the control and GS group with respect to any of the SF-36 subscales.

Our study has some important limitations. The small number of participants did not allow us to perform GS subgroups analyses of discrete GI symptoms, extra-intestinal manifestations and other related factors, which may play a role in the perception of gluten sensitivity. Preferably a gluten-free control would have been included, but this choice was not made because of the inability to blind gluten-free bread against wheat-based breads.

It is possible that other factors in bread such as FODMAP or the nongluten ATI, which were not targeted by the enzyme, are responsible for the observed effects. In support of FODMAP as the causative agent, a recently published study investigating the effects of gluten (without fructan) and fructan (without gluten) on GI symptoms in individuals intolerant to wheat, rye or barley reported that fructan caused an increase in GI symptoms compared with the placebo control ${ }^{(48)}$. It would have been beneficial to control for FODMAP intolerances. However, because these resilient carbohydrates occur not just in wheat but in all kind of foods, such interventions involve individual consultation with a specialist dietitian, who advises on 4-8-week exclusion of foods high in FODMAP, followed by graded reintroduction. Such an approach was beyond the scope of this study and therefore a role for FODMAP in the bread cannot be ruled out.

The lack of unambiguous biomarkers also makes it difficult to confirm the true clinical status of the GS group with respect to NCGS. 
In conclusion, the proline-specific ANPEP enzyme lowers the immunogenic content of gluten by approximately $40 \%$ and can be used in the production of wheat products comparable to standard gluten content products in terms of taste, texture and appearance. Only consumption of reference and biscuit flour bread increased GI symptoms in the GS subjects, compared with their habitual diet. However, the $40 \%$ gluten reduction in the ANPEP treated bread showed no treatment effect compared with the other test breads. This may be due to levels of gluten in ANPEP bread being insufficiently reduced to keep the GI symptoms to pre-treatment levels.

Another explanation might be that gluten is not the main driver of the symptoms in this population - a gluten-free or wheat-free diet will not solve the GI symptoms if FODMAP, ATI or other (presently unknown) factors are the main causes of symptoms. This will clearly need to be considered in future studies.

The increase in GI symptoms after consumption of the test breads may also be a consequence of a nocebo effect in the GS population. Self-defined sensitive to gluten individuals who experience mild discomfort when consuming gluten, adopt a low-gluten lifestyle as opposed to following a strict GFD. This approach helps to reduce the GI symptoms severity to tolerable levels. Consumption of wheat bread increases symptoms in the GS subjects in comparison with their habitual diet. In comparison with healthy controls, the GS subjects have a favourable CVD lipid profile and modified haematological profile. Finally, a low-gluten lifestyle was shown to have no effect on the quality of life, fatigue or mental state of the GS population.

\section{Acknowledgements}

The authors are grateful for the support of the staff from the Rowett Institute Human Nutrition Unit

The present study was supported by a grant (Bakery Products for Non-Coeliac Gluten sensitive Consumers) from Innovate UK (101740). The funder Innovate UK had no role in the design, analysis or writing of this article. Warburton Ltd. provided the experimental bread. DSM Food Specialities B.V. provided the protease ANPEP. The study is registered under the Clinical Trials Identifier: NCT02308397.

N. H. proposed the study concept. N. H. and D. R. designed research. D. R. recruited the subjects and carried out the intervention, laboratory analysis, data collection, collation and prepared the manuscript. M. C. and K. M. M. helped with recruitment. G. C. performed the analysis of the breads. G. H. and D. R. analysed the data. N. H. had primary responsibility for final content. All authors read and approved the final manuscript.

The authors declare that there are no conflicts of interest.

\section{References}

1. Sapone A, Bai JC, Ciacci C, et al. (2012) Spectrum of glutenrelated disorders: Consensus on new nomenclature and classification. BMC Medicine 10, 13.

2. di Sabatino A \& Corazza GR (2012) Nonceliac gluten sensitivity: Sense or sensibility? Ann Intern Med 156, 309-311.
3. Marsh MN (1992) Gluten, major histocompatibility complex, and the small intestine. A molecular and immunobiologic approach to the spectrum of gluten sensitivity ('celiac sprue'). Gastroenterology 102, 330-354.

4. Biesiekierski JR, Newnham ED, Irving PM, et al. (2011) Gluten causes gastrointestinal symptoms in subjects without celiac disease: a double-blind randomized placebo-controlled trial. Am J Gastroenterol 106, 508-514.

5. Carroccio A, Mansueto P, Iacono G, et al. (2012) Non-celiac wheat sensitivity diagnosed by double-blind placebocontrolled challenge: exploring a new clinical entity. Am J Gastroenterol 107, 1898-1906.

6. Biesiekierski JR, Peters SL, Newnham ED, et al. (2013) No effects of gluten in patients with self-reported non-celiac gluten sensitivity after dietary reduction of fermentable, poorly absorbed, short-chain carbohydrates. Gastroenterology $\mathbf{1 4 5}$, 320-328.e3.

7. Di Sabatino A, et al. (2015) Small amounts of gluten in subjects with suspected nonceliac gluten sensitivity: a randomized, double-blind, placebo-controlled, cross-over trial. Clin Gastroenterol Hepatol 13, 1604-1612.

8. Junker Y, Zeissig S, Kim SJ, et al. (2012) Wheat amylase trypsin inhibitors drive intestinal inflammation via activation of toll-like receptor 4. J Exp Med 209, 2395-2408.

9. Shan L, Molberg $\varnothing$, Parrot I, et al. (2002) Structural basis for gluten intolerance in Celiac Sprue. Science 297, 2275-2279.

10. Wieser H (1995) 1 The precipitating factor in coeliac disease. Baillieres Clin Gastroenterol 9, 191-207.

11. Hausch F, Shan L, Santiago NA, et al. (2002) Intestinal digestive resistance of immunodominant gliadin peptides. Am J Physiol Gastrointest Liver Physiol 283, G996-G1003.

12. Stepniak D, Spaenij-Dekking L, Mitea C, et al. (2006) Highly efficient gluten degradation with a newly identified prolyl endoprotease: implications for celiac disease. Am J Physiol Gastrointest Liver Physiol 291, G621-G629.

13. Mitea C, Havenaar R, Wouter Drijfhout J, et al. (2008) Efficient degradation of gluten by a prolyl endoprotease in a gastrointestinal model: Implications for coeliac disease. Gut 57, 25-32.

14. Salden BN, Monserrat V, Troost FJ, et al. (2015) Randomised clinical study: Aspergillus niger-derived enzyme digests gluten in the stomach of healthy volunteers. Aliment Pharmacol Ther 42, 273-285

15. Chamberlain N, Collins TH \& Elton GAH (1962) The Chorleywood bread process. Bakers Dig 36, 52-53.

16. Mena MC, Lombardía M, Hernando A, et al. (2012) Comprehensive analysis of gluten in processed foods using a new extraction method and a competitive ELISA based on the R5 antibody. Talanta 91, 33-40.

17. Biagi F, Andrealli A, Bianchi PI, et al. (2009) A gluten-free diet score to evaluate dietary compliance in patients with coeliac disease. Br J Nutr 102, 882-887.

18. Biagi F, Bianchi PI, Marchese A, et al. (2012) A score that verifies adherence to a gluten-free diet: a cross-sectional, multicentre validation in real clinical life. Br J Nutr 108, 1884-1888.

19. Ware JE, Snow K, Kosinski M, et al. (editors) (1983) SF-36 Health Survey: Manual and Interpretation Guide. Boston, MA: New England Medical Center, Health Institute.

20. Baecke JAH, Burema J \& Frijters JER (1982) A short questionnaire for the measurement of habitual physical activity in epidemiological studies. Am J Clin Nutr 36, 936-942.

21. Lovibond SH \& Lovibond PF (editors) (1995) Manual for the Depression Anxiety Stress Scales, 2nd ed. Sydney: Psychology Foundation of Australia.

22. Fisk JD \& Doble SE (2002) Construction and validation of a fatigue impact scale for daily administration (D-FIS). Qual Life Res 11, 263-272. 
23. Spiller RC (1999) Problems and challenges in the design of irritable bowel syndrome clinical trials: experience from published trials. Am J Med 107, 91-97.

24. Kasarda DD, Nimho CC \& Kohler GO (1971) Proteins and the amino acid composition of wheat fractions. In Wheat Chemistry and Technology, pp. 227-299 [V Pomeranz, editor]. St. Paul, MN: American Association of Cereal Chemists.

25. The International Association of Plant Bakers (2015) AIBI Bread Market Report. http://www.aibi.eu/wp-content/ uploads/draft-AIBI-Bread-Market-report-2013.pdf (accessed March 2016).

26. Scottish Government (2012) Food and drink in Scotland: key facts. Healthier - diet and nutrition. http://www.gov.scot/ Publications/2012/03/3347/9 (accessed March 2016).

27. Zanini B, Baschè R, Ferraresi A, et al. (2015) Randomised clinical study: gluten challenge induces symptom recurrence in only a minority of patients who meet clinical criteria for noncoeliac gluten sensitivity. Aliment Pharmacol Ther 42, 968-976.

28. National Institute for Health and Care Excellence (NICE) (2015) Coeliac disease: recognition, assessment and management. Version 2. https://www.nice.org.uk/guidance/ng20 (accessed April 2016).

29. Volta U, Tovoli F, Cicola R, et al. (2012) Serological tests in gluten sensitivity (nonceliac gluten intolerance). J Clin Gastroenterol 46, 680-685.

30. Stapel SO, Asero R, Ballmer-Weber BK, et al. (2008) Testing for IgG4 against foods is not recommended as a diagnostic tool: EAACI Task Force Report. Allergy 63, 793-796.

31. Wang W, Uzzau S, Goldblum SE, et al. (2000) Human zonulin, a potential modulator of intestinal tight junctions. J Cell Sci 113, 4435-4440.

32. Fasano A (2012) Zonulin, regulation of tight junctions, and autoimmune diseases. Ann N Y Acad Sci 1258, 25-33.

33. Smecuol E, Sugai E, Niveloni S, et al. (2005) Permeability, zonulin production, and enteropathy in dermatitis herpetiformis. Clin Gastroenterol Hepatol 3, 335-341.

34. West J, Logan RFA, Hill PG, et al. (2003) Seroprevalence, correlates, and characteristics of undetected coeliac disease in England. Gut 52, 960-965.

35. Lewis NR, Sanders DS, Logan RFA, et al. (2009) Short communication: cholesterol profile in people with newly diagnosed coeliac disease: A comparison with the general population and changes following treatment. BrJ Nutr 102, 509-513.
36. Baydoun A, Maakaron JE, Halawi H, et al. (2012) Hematological manifestations of celiac disease. Scand J Gastroenterol 47, 1401-1411.

37. Bottaro G, Cataldo F, Rotolo N, et al. (1999) The clinical pattern of subclinical/silent celiac disease: an analysis on 1026 consecutive cases. Am J Gastroenterol 94, 691-696.

38. Swinson CM \& Levi AJ (1980) Is coeliac disease underdiagnosed? Br Med J 281, 1258-1260.

39. Logan RFA, Tucker G, Rifkind EA, et al. (1983) Changes in clinical features of coeliac disease in adults in Edinburgh and the Lothians 1960-79. Br Med J 286, 95-97.

40. Bessman JD, Gilmer PR Jr \& Gardner FH (1983) Improved classification of anemias by MCV and RDW. Am J Clin Pathol 80, 322-326.

41. Rostami K, al Dulaimisss D, Nejad MR, et al. (2010) Microscopic enteritis and pathomechanism of malabsorption. Auto Immun Highlights 1, 37-38.

42. Yüksel O, Helvac K, Basar O, et al. (2009) An overlooked indicator of disease activity in ulcerative colitis: mean platelet volume. Platelets 20, 277-281.

43. Endler G, Klimesch A, Sunder-Plassmann H, et al. (2002) Mean platelet volume is an independent risk factor for myocardial infarction but not for coronary artery disease. Br J Haematol 117, 399-404.

44. Berthouze SE, Dumoulin C \& Reynes E (2016) Fatigue and physical activity: a decisive issue in the chronical disease care pathways. Ann Phys Rehabil Med 59S, e51-e52.

45. Di Sabatino A, Volta U, Salvatore C, et al. (2015) Small amounts of gluten in subjects with suspected nonceliac gluten sensitivity: a randomized, double-blind, placebocontrolled, cross-over trial. Clin Gastroenterol Hepatol 13, 1604-1612.

46. Peters SL, Biesiekierski JR, Yelland GW, et al. (2014) Randomised clinical trial: gluten may cause depression in subjects with non-coeliac gluten sensitivity - an exploratory clinical study. Aliment Pharmacol Ther 39, 1104-1112.

47. Brottveit M, Vandvik PO, Wojniusz S, et al. (2012) Absence of somatization in non-coeliac gluten sensitivity. Scand J Gastroenterol 47, 770-777.

48. Skodje GI, Sarna VK, Minelle IH, et al. (2017) Fructan, rather than gluten, induces symptoms in patients with self-reported non-celiac gluten sensitivity. Gastroenterology (Epublication ahead of print version November 2017). 